\title{
On the validity of the ambipolar diffusion assumption in the polar mesopause region
}

\author{
A. P. Ballinger ${ }^{1}$, P. B. Chilson ${ }^{2}$, R. D. Palmer ${ }^{2}$, and N. J. Mitchell ${ }^{3}$ \\ ${ }^{1}$ Program in Atmospheric and Oceanic Sciences, Princeton University, Princeton, NJ, USA \\ ${ }^{2}$ School of Meteorology and Atmospheric Radar Research Center, University of Oklahoma, Norman, OK, USA \\ ${ }^{3}$ Department of Electronic \& Electrical Engineering, University of Bath, Bath, UK
}

Received: 26 June 2008 - Revised: 30 October 2008 - Accepted: 31 October 2008 - Published: 6 November 2008

\begin{abstract}
The decay of underdense meteor trails in the polar mesopause region is thought to be predominantly due to ambipolar diffusion, a process governed by the ambient temperature and pressure. Hence, observations of meteor decay times have been used to indirectly measure the temperature of the mesopause region. Using meteor observations from a SKiYMET radar in northern Sweden during 2005, this study found that weaker meteor trails have shorter decay times (on average) than relatively stronger trails. This suggests that processes other than ambipolar diffusion can play a signicant role in trail diffusion. One particular mechanism, namely electron-ion recombination, is explored. This process is dependent on the initial electron density within the meteor trail, and can lead to a disproportionate reduction in decay time, depending on the strength of the meteor.
\end{abstract}

Keywords. Atmospheric composition and structure (Pressure, density, and temperature; Instruments and techniques)

\section{Introduction and background}

At any location during any given day, many thousands of meteors enter our Earth's upper atmosphere. The frequency of incoming meteors fluctuates but generally follows a wellunderstood diurnal and seasonal cycle. Most of the meteors ablate as they interact with the increasingly dense air molecules, leaving an ionized plasma trail in their wake. A meteor radar is able to detect these short-lived trails (herein referred to as "meteor echoes"), enabling certain useful parameters, such as drift velocity, decay times, etc., to be estimated.

Consider the idealized case where the radius of a meteor trail is much smaller than the radar wavelength, and the effects of diffusion can be ignored. Further, assume the trail is

Correspondence to: A. P. Ballinger

(aballing@ princeton.edu) "underdense", meaning each electron in a meteor trail scatters independently, with a scattering cross-section given by

$\sigma_{e}=\frac{\mu_{0}^{2} e^{4}}{16 \pi^{2} m^{2}}$,

where $\mu_{0}, m$ and $e$ are the magnetic permeability of air, electron mass, and electron charge, respectively.

By assuming ambipolar diffusion is the predominant mechanism by which the meteor echo decays, the backscattered power will fall off from an initial value of $P_{0}$ according to

$P(t)=P_{0} \exp \left[-\frac{32 \pi^{2} D_{a} t}{\lambda^{2}}\right]$,

where $D_{a}$ is the "ambipolar diffusion coefficient", and $t$ is the time after the initial peak power (Chilson et al., 1996). Defining a "decay time", $\tau_{1 / 2}$, as the time taken for the power to drop to half the peak, the ambipolar diffusion coefficient can be estimated from the meteor echo decay time by

$D_{a}=\frac{\lambda^{2} \ln 2}{16 \pi^{2} \tau_{1 / 2}}$.

This ambipolar diffusion coefficient is dependent on the atmospheric temperature, $T$, and pressure, $p$, through the relation

$D_{a}=K_{\mathrm{amb}} \frac{T^{2}}{p}$,

where $K_{\mathrm{amb}}$ is a constant (Jones and Jones, 1990; Jones, 1995; Chilson et al., 1996; Hocking et al., 1997). Hence, if either $T$ or $p$ is known, the other parameter can be deduced once $D_{a}$ has been determined from the meteor echo decay times. Other methods have also been developed, primarily to estimate temperature (e.g. Hocking et al., 1997), that do not require observed (or modeled) pressure, but instead use the vertical profile of meteor decay times. Each

Published by Copernicus Publications on behalf of the European Geosciences Union. 


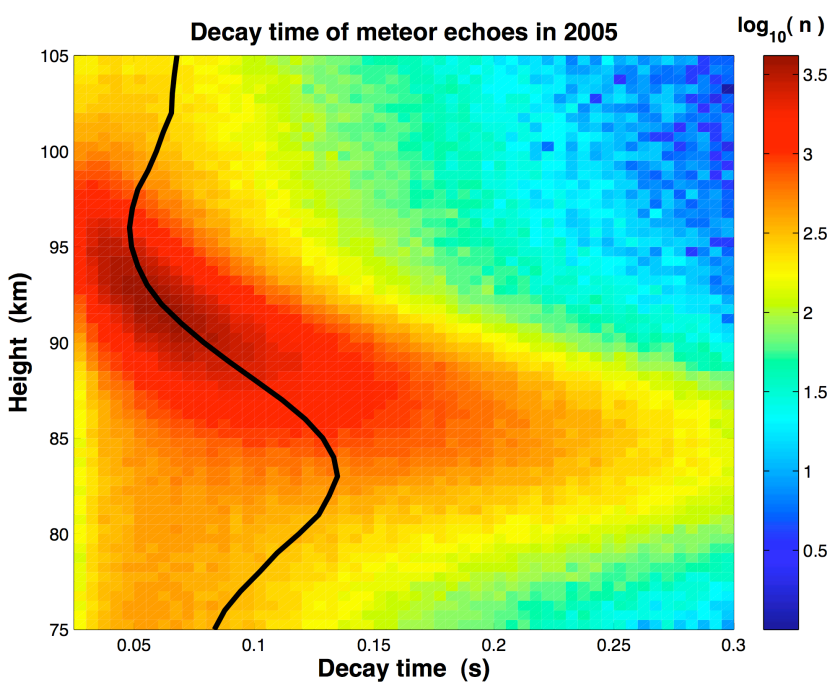

Fig. 1. Decay time versus height of all meteors during 2005. Color shading indicates the number of meteors, $n$ (per $500 \mathrm{~m} \times 5 \mathrm{~ms}$ window). The solid line indicates the mean decay time.

method of temperature estimation that uses ablating meteors requires the assumption that ambipolar diffusion alone governs the decay of the underdense meteor echoes. However, alternative mechanisms exist, which can also affect the decay rate (Dyrud et al., 2001; Havnes and Sigernes, 2005; Dimant and Oppenheim, 2006a,b; Holdsworth et al., 2006).

\section{Instrumentation and observations}

In order to investigate the decay of meteor echoes, observations taken from a Very-High-Frequency (VHF) meteor radar located at Esrange, near Kiruna, in northern Sweden, during 2005 were analyzed. The All-Sky Interferometric Meteor Radar (SKiYMET) system is a multi-channel coherent receiver pulsed radar capable of observing a wide range of parameters through the detection and analysis of faint meteors (see Hocking et al., 2001, for details).

The radar transmits at $32.5 \mathrm{MHz}(\lambda=9.23 \mathrm{~m})$, with a typical pulse repetition frequency (PRF) of $2143 \mathrm{kHz}$. A pulse length of $13.3 \mu \mathrm{s}$ corresponds to a relatively poor range resolution of $2 \mathrm{~km}$, which leads to some uncertainty as to the altitude of any given meteor trail. However, the coarser resolution means that a meteor trail is most likely fully contained within a range gate, which is important in building accurate statistics. So the uncertainty in the height measurement is considered small when averaged over a large number of meteors.

The PRF produces an aliasing range of $70 \mathrm{~km}$. However, since most meteors ablate at a height of $90 \mathrm{~km}( \pm 20 \mathrm{~km})$, the meteor signals detected are at least second-trip echoes, depending on the zenith angle and range of the individual meteor. Over the course of 2005, more than 3.9 million meteors were observed over Esrange, averaging over 10000 per day. Over half of these were rejected in order to ensure that only the most reliable meteor signals, and corresponding decay times, were retained (see Ballinger, 2007, for further details of the filtering process).

\section{Data analysis and results}

\subsection{Meteor decay times}

In order to construct a vertical profile of the average meteor decay times, a representative decay time for each height was determined. Firstly, the incoming meteors over a certain time period were grouped into height "bins" of $1 \mathrm{~km}$. This bin width allowed sufficient vertical resolution, while still ensuring a large number of meteors was in each group. The distribution of decay times (within each height interval) is normal for the logarithm transformed values, hence the "geometric" mean $(\bar{x})$ and standard deviation $(\sigma)$ can be calculated by

$\bar{x}=\exp [\overline{\log X}]$

$\sigma=\exp \left[\sigma_{\log X}\right]$

where $X$ is the log-normally distributed variable (Aitchison and Brown, 1957). A confidence interval (in the position of the mean) is given by

$\frac{\bar{x}}{\sigma^{\frac{z_{\alpha / 2}}{\sqrt{n}}}}<\mu<\bar{x} \sigma^{\frac{z_{\alpha / 2}}{\sqrt{n}}}$,

for a sample of $n$ meteors (Miller and Freund, 1977). Here, $\mu$ represents the actual mean of the distribution, with a $1-\alpha$ probability of lying within the bounds of the confidence interval, and $z_{\alpha / 2}$ is such that the area under a normal curve to its right equals $\alpha / 2$. For instance, to find the $95 \%$ confidence interval $(\alpha=0.05)$ in the position of the mean, one would set $z_{\alpha / 2}=1.96$; for $99 \%$ confidence interval $(\alpha=0.01)$, one would set $z_{\alpha / 2}=2.947$ (Miller and Freund, 1977).

\subsection{Height profile of decay times}

The annual mean decay time vertical profile for 2005 is shown in Fig. 1. The number of meteors (color shading) reflects the height distribution of incoming meteors, with the majority of meteors falling between $80 \mathrm{~km}$ and $100 \mathrm{~km}$. The $99 \%$ confidence intervals are not shown since they fall within the thickness of the line that plots the mean decay time profile, indicating the general features of the vertical profile are reliable.

The vertical decay time profile is characterized by a lower maximum at approximately $83 \mathrm{~km}$, with decay time decreasing with altitude above this level, until an upper minimum at approximately $96 \mathrm{~km}$. The meteor decay times throughout this region are assumed to be governed by ambipolar diffusion (e.g. Jones, 1975; Jones and Jones, 1990; Hocking et al., 
1997), with decay time being proportional to pressure (decreasing with altitude). The vertical profile of decay time below $83 \mathrm{~km}$, and above $96 \mathrm{~km}$, can be described as "kickback regions", where the decay time appears to increase with altitude (for a similar result, see Fig. 1 in Hall et al., 2005). Although the number of meteors is significantly less in these regions, it appears these features are real, and have been briefly discussed by others (e.g. Dyrud et al., 2001; Hall, 2002; Hall et al., 2005).

Dyrud et al. (2001) attribute the upper level increase in diffusion (decrease in decay time) to gradient drift FarleyBuneman (GDFB) instability (Fejer et al., 1975), that develops where the trail density gradient and electric field are largest. Above approximately $100 \mathrm{~km}$ (perhaps as much as $5 \mathrm{~km}$ lower at polar latitudes), collisions dominate ion motion causing them to diffuse out of the trail. The electrons are unable to follow the ions, creating an electric field perpendicular to the meteor trail. GDFB instabilities can grow, leading to anomalous diffusion that exceeds the ambipolar diffusion rate by an order of magnitude (Dyrud et al., 2001). At lower altitudes (below $96 \mathrm{~km}$ ), electrons diffuse faster than ions, reversing the electric field and damping any GDFB instability.

The reason for the lower "kickback" is more puzzling, with only brief discussion in the literature to date. Hall (2002) acknowledged that this feature is common, and that diffusion only rarely continues to decrease (decay time increase) at altitudes lower than $80-85 \mathrm{~km}$. This is contrary to predictions from ambipolar diffusion theory (Eq. 4), suggesting another process (or other processes) contribute at these altitudes. The confidence intervals defining the position of the mean decay time profile are sufficiently narrow to rule out a statistical-averaging effect due to the relatively low number of meteors in this height region.

\subsection{Decay times within the mesopause region}

As previously mentioned, the decay time of meteor echoes near the mesopause is thought to be predominantly governed by ambipolar diffusion (e.g. Jones, 1975; Jones and Jones, 1990; Hocking et al., 1997). Therefore, the remainder of this analysis will focus on the atmosphere in the height range of 80 to $90 \mathrm{~km}$. It has been proposed that processes other than ambipolar diffusion can have a detectable influence on meteor decay times throughout the mesopause region (e.g. Dyrud et al., 2001; Havnes and Sigernes, 2005; Dimant and Oppenheim, 2006a,b; Holdsworth et al., 2006). By ignoring these effects, one might inadvertently overestimate the ambipolar diffusion coefficient, which could have important consequences for temperature estimation, and thus deserves further investigation. In particular, we consider electron-ion recombination (also loosely referred to as electron "absorption") and the potential impact that it could have on meteor decay times.

It has been suggested by Havnes and Sigernes (2005) that charged particles should have a more pronounced effect on

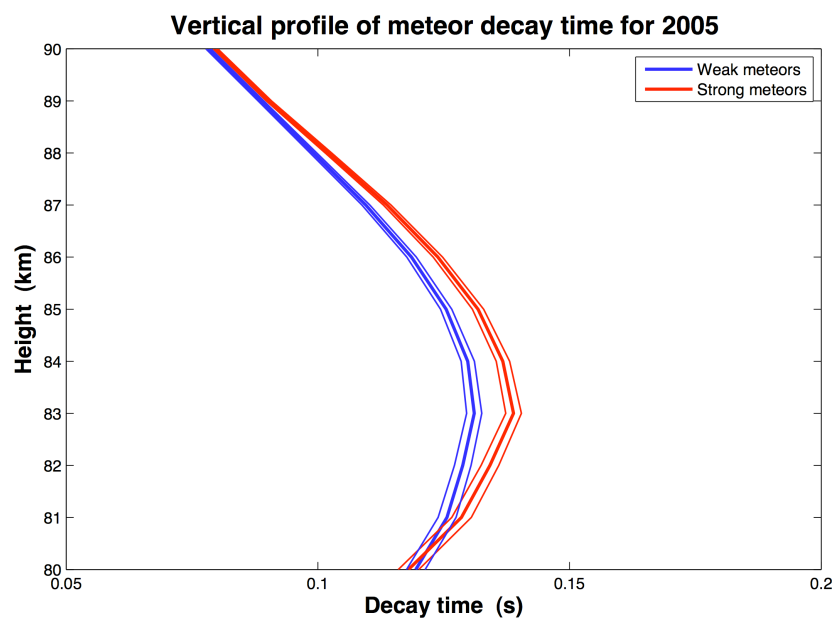

Fig. 2. Vertical profiles of mean decay time for 2005. The profile of weak meteors $(\mathrm{SNR}<12 \mathrm{~dB})$ is shown in blue; strong meteors $(\mathrm{SNR} \geq 12 \mathrm{~dB})$ in red. The thin lines either side of the mean profiles indicate the $99 \%$ confidence interval bounds.

meteor trails with relatively weak echo powers, compared to stronger ones. The term "charged particles" is used here to describe the charged species that are quasi-continually present in the mesopause region. These particles arise from a variety of processes such as ionization from solar radiation, particle precipitation from the lower thermosphere, and the ablation of meteors (Brasseur and Solomon, 1986). The mechanism proposed by Havnes and Sigernes (2005) follows from an assumption that a given concentration of positive ions in the background environment is available for absorbing a certain number of electrons in a rapidly expanding and diffusing meteor trail. The number of electrons removed from the trail in this way is expected to be relatively independent of the initial density of electrons in the trail (i.e. the "echo strength" of the trail). Hence, electron absorption will tend to remove a greater fraction of the initial electrons from within a weak meteor trail, compared to the fraction removed from within a relatively stronger trail.

In order to investigate the potential impact of electronion recombination on the decay rate of meteor echoes, incoming meteors were divided into two groups of approximately the same number, based on the initial trail strength. Meteor echoes with a signal-to-noise ratio $(\mathrm{SNR})<12 \mathrm{~dB}$ were deemed weak, while echoes with a $S N R \geq 12 \mathrm{~dB}$ were deemed strong. Note that this is not an absolute categorization, but rather a method of dividing the total echoes into approximately equal numbers of "relatively weaker" and "relatively stronger" meteor echoes. As per the hypothesis of Havnes and Sigernes (2005), the decay time of the weaker echoes should be more greatly affected by the presence of charged particles. Results of the analysis are shown in Fig. 2. A significant reduction in decay time is evident throughout the range $\sim 82-88 \mathrm{~km}$, with a maximum reduction of approximately $0.01 \mathrm{~s}(\sim 10 \%)$ at $83 \mathrm{~km}$. 


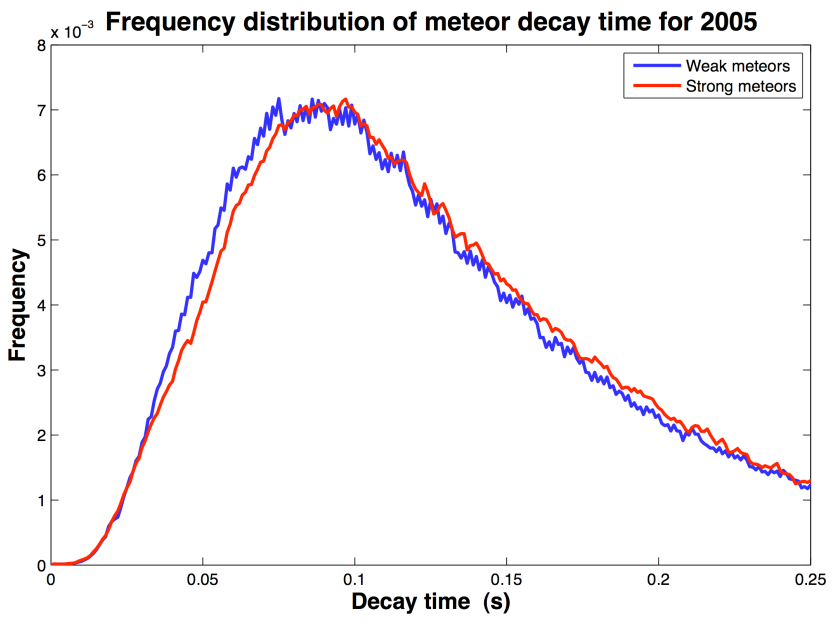

Fig. 3. Frequency distributions of meteor decay times for 2005. The distribution of weak meteors $(\mathrm{SNR}<12 \mathrm{~dB})$ is shown in blue; strong meteors $(\mathrm{SNR} \geq 12 \mathrm{~dB})$ in red.

The frequency distribution of decay time for weak and strong meteors for incoming meteors at heights between $80 \mathrm{~km}$ and $90 \mathrm{~km}$ was also calculated and is shown in Fig. 3. There appears to be a pronounced shift towards lower decay times for the weaker echoes. This reduction is apparent across most decay times, showing little evidence for reducing short or long decay times preferentially.

The results found here can be compared against the calculations presented in Havnes and Sigernes (2005). They considered three different scenarios involving dust particles of different radii $r_{d}$, charge numbers $Z_{d}$, and number densities $n_{d}$. Their Fig. 1 shows the anticipated reduction in decay times (in \%) for case 1: $r_{d} \approx 6 \mathrm{~nm}, N_{d}=+1$, and $n_{d}=5 \times 10^{9} \mathrm{~m}^{-3}$ (aerosols at a height of $85 \mathrm{~km}$ ); case 2 : $r_{d} \approx 6 \mathrm{~nm}, N_{d}=+1$, and $n_{d}=4 \times 10^{10} \mathrm{~m}^{-3}$ (aerosols at a height of $90 \mathrm{~km})$; and case $3: r_{d} \approx 15 \mathrm{~nm}, N_{d}=0$, and $n_{d}=4 \times 10^{9} \mathrm{~m}^{-3}$ (night conditions with little or no particle precipitation). Assuming that little reduction in the decay time occurs for the strong meteor cases shown in Figs. 2 and 3 then the results shown in Fig. 1 of Havnes and Sigernes (2005) can be compared to the reductions in decay time found here for the weak meteor cases. A $10 \%$ reduction in decay time would correspond to a value for the infinite crosssectional slice of a meteor trail of unit thickness of approximately $4 \times 10^{11}, 5 \times 10^{11}$, and $4 \times 10^{12}$ for cases 1,2 , and 3 , respectively. Note that the calculations of Havnes and Sigernes (2005) were made for a radar wavelength $\lambda=8 \mathrm{~m}(\lambda=9.23 \mathrm{~m}$ for the measurements presented here). All that can be said at this point is that these values are certainly within the range of expected values for typical meteor trails.

\section{Conclusions}

Meteor radars are routinely used around the world to measure upper atmospheric parameters such as the magnitude and direction of the wind and the temperature. Generally meteor radars are capable of producing reliable daily estimates of the mean atmospheric temperature near the mesopause using the methods outlined in Hocking et al. (1997, 2001). However, one should exercise caution when invoking the necessary assumption that the observed meteor echo decay times are primarily governed by ambipolar diffusion. Other factors can contribute to the decay rate, which would tend to bias the temperature estimates to larger values. It was shown that some mechanism was acting to reduce the meteor echo decay times in the summer polar mesopause region and that this mechanism acted preferentially on weaker echoes. One possible explanation for this behavior can be found in electronion recombination (Havnes and Sigernes, 2005). Further studies are needed to better quantify the potential impact of electron-ion recombination on meteor echo decay and to determine the extent to which it could impact different methods of temperature determination.

Acknowledgements. This research has been funded in part by the National Science Foundation, through a CEDAR grant (ATM0532707). The data used in this research were made available by the Swedish Institute of Space Physics (IRF) and the Swedish Space Corporation - Esrange. Their continued support of this research is appreciated. Helpful comments from Wayne Hocking during the preparation of this paper are also appreciated.

Topical Editor U.-P. Hoppe thanks L. Dyrud for his help in evaluating this paper

\section{References}

Aitchison, J. and Brown, J. A. C.: The lognormal distribution, Cambridge University Press, Cambridge, 1957.

Ballinger, A.: Radio-wave scatter and dynamical processes in the polar mesopause region observed using MST and meteor radars, MSc Thesis, University of Oklahoma, 2007.

Brasseur, G. and Solomon, S.: Aeronomy of the middle atmosphere, D. Reidel Publishing Company, Dordrecht, Holland, second edn., 1986.

Chilson, P. B., Czechowsky, P., and Schmidt, G.: A comparison of ambipolar diffusion coefficients in meteor trains using VHF radar and UV lidar, Geophys. Res. Lett., 23, 2745-2748, 1996.

Dimant, Y. S. and Oppenheim, M. M.: Meteor trail diffusion: 1. Simulations, J. Geophys. Res., 111, A12312, doi:10.1029/2006JA011797, 2006a.

Dimant, Y. S. and Oppenheim, M. M.: Meteor trail diffusion: 2. Analytical theory, J. Geophys. Res., 111, A12313, doi:10.1029/2006JA011798, 2006b.

Dyrud, L. P., Oppenheim, M. M., and vom Endt, A. F.: The anomalous diffusion of meteor trails, Geophys. Res. Lett., 28, 27752778, 2001.

Fejer, B. G., Farley, D. T., Balsley, B. B., and Woodman, R. F.: Vertical structure of the VHF backscattering region in the equatorial 
electrojet and the gradient drift instability, J. Geophys. Res., 80, 1313-1324, 1975.

Hall, C. M.: On the influence of neutral turbulence on ambipolar diffusivities deduced from meteor trail expansion, Ann. Geophys., 20, 1857-1862, 2002,

http://www.ann-geophys.net/20/1857/2002/.

Hall, C. M., Aso, T., Tsutsumi, M., Nozawa, S., Manson, A., and Meek, C. E.: Testing the hypothesis of the influence of neutral turbulence on the deduction of ambipolar diffusivities from meteor trail expansion, Ann. Geophys., 23, 1071-1073, 2005, http://www.ann-geophys.net/23/1071/2005/.

Havnes, O. and Sigernes, F.: On the influence of background dust on radar scattering from meteor trails, J. Atmos. Sol. Terr. Phys., 67, 659-664, 2005.

Hocking, W. K., Thayaparan, T., and Jones, J.: Meteor decay times and their use in determining a diagnostic mesospheric temperature-pressure parameter: Methodology and one year of data, Geophys. Res. Lett., 24, 2977-2980, 1997.
Hocking, W. K., Fuller, B., and Vandepeer, B.: Real-time determination of meteor-related parameters utilizing modern digital technology, J. Atmos. Sol. Terr. Phys., 63, 155-169, 2001.

Holdsworth, D. A., Morris, R. J., Murphy, D. J., Reid, I. M., Burns, G. B., and French, W. J. R.: Antarctic mesospheric temperature estimation using the Davis mesospherestratosphere-troposphere radar, J. Geophys. Res., 111, D05108, doi:10.1029/2005JD006589, 2006.

Jones, J.: On the decay of underdense radio meteor echoes, Mon. Not. Roy. Astr. Soc., 173, 637-647, 1975.

Jones, W.: The decay of radar echoes from meteors with a particular reference to their use in the determination of temperature fluctuations near the mesopause, Ann. Geophys., 13, 1104-1106, 1995, http://www.ann-geophys.net/13/1104/1995/.

Jones, W. and Jones, J.: Ionic diffusion in meteor trails, J. Atmos. Terr. Phys., 52, 185-191, 1990.

Miller, E. and Freund, J. E.: Probability and statistics for engineers, Prentice-Hall, Inc., New Jersey, second edn., 1977. 